\title{
Inhibition of microRNA-30a prevents puromycin aminonucleoside-induced podocytic apoptosis by upregulating the glucocorticoid receptor $\alpha$
}

\author{
HUA XIE ${ }^{1,2}$, HONG-LI LIN ${ }^{2}$, NAN WANG ${ }^{2}$, YAN-LING SUN ${ }^{2}$, \\ $\mathrm{YU} \mathrm{KAN}^{2}$, HUI GUO ${ }^{2}$, JI-LIN CHEN ${ }^{2}$ and MING FANG ${ }^{2}$ \\ ${ }^{1}$ Graduate School of Dalian Medical University, Dalian, Liaoning 116044; ${ }^{2}$ Department of Nephrology, \\ The First Affiliated Hospital of Dalian Medical University, Dalian, Liaoning 116011, P.R. China
}

Received October 18, 2014; Accepted July 28, 2015

DOI: $10.3892 / \mathrm{mmr} .2015 .4226$

\begin{abstract}
It is well-established that steroid-resistant nephrotic syndrome commonly involves podocytic injury, however, the underlying mechanism remains to be elucidated. Previous studies have demonstrated that glucocorticoids enhance podocytic recovery predominantly through the functional isoform, glucocorticoid receptor (GR) $\alpha$. Our previous study demonstrated the reduced expression of GR $\alpha$ in podocytes from patients with steroid-resistant nephrotic syndrome compared with those with steroid-sensitive syndromes, which suggested that microRNAs (miRNAs) may be a potential therapeutic target in the modulation of steroid sensitivity. The present study screened miRNAs in murine injured podocytes by microarray and identified 10 miRNAs significantly upregulated, including miR-30a, miR-30d, miR-100, miR181c, miR-5099, miR-3535, miR-140-3p, miR-148-3p and miR-103-3p. Bioinformatic target prediction indicated that the GR was a candidate target gene of miR-30a. The data also indicated that miR-30a negatively regulated GR $\alpha$ in normal and injured podocytes induced by puromycin aminonucleoside (PAN). In addition, the inhibition of miR-30a prevented podocytic apoptosis induced by PAN. However, luciferase reporter assay data suggested an indirect effect on the transcriptional activity of GR $\alpha$. The present study indicated that silencing of miR-30a may improve steroid sensitivity in injured podocytes, although the mechanism cannot be explained by conventional means and remains to be elucidated.
\end{abstract}

Correspondence to: Dr Hong-Li Lin, Department of Nephrology, The First Affiliated Hospital of Dalian Medical University, 222 Zhongshan Road, Dalian, Liaoning 116011, P.R. China

E-mail: hllin@dlmedu.edu.cn

Key words: podocyte, microRNA-30a, apoptosis, glucocorticoid receptor $\alpha$

\section{Introduction}

Podocytes are critical in maintaining the integrity of the glomerular filtration barrier. Acquired proteinuric glomerulopathies, including minimal change disease and focal segmental glomerulosclerosis, commonly exhibit abnormalities in podocytic phenotypes, including effacement and apoptosis $(1,2)$. Glucocorticoids, which are the primary therapy for the treatment of proteinuric glomerulopathies, exert profound effects through their interaction with specific glucorticoid receptors (GRs) (3). GRs are abundantly expressed in human and mouse podocytes $(4,5)$, and exist in different isoforms as a result of alternative splicing. GR $\alpha$ is the functionally active isoform located in the cytoplasm. Glucocorticoids are reported to induce recovery in podocyte injuries predominantly via two GR $\alpha$-mediated mechanisms. Genetic regulation occurs following the binding of glucocorticoids at low doses $(0.5 \mathrm{mg} / \mathrm{kg} / \mathrm{day})$, resulting in the activation and translocation of GR $\alpha$ into the nucleus. Subsequently, activated GR $\alpha$ binds at the glucocorticoid response elements within the genome to mediate the direct regulation of target genes leading to anti-inflammatory and immunosuppressive effects (5). The alternative mechanism involves the direct effect of glucocorticoids on podocyte-expressed GR $\alpha$ (4), which reduces podocytic apoptosis and promotes actin aggregation (6). Therefore, GR $\alpha$ regulation is a crucial determinant of steroid efficacy.

Our previous study demonstrated a reduced expression of GR $\alpha$ in podocytes from patients with steroid-resistant idiopathic nephrotic syndrome compared with steroid-sensitive patients (7). Therefore, upregulation of the GR $\alpha$ may be a promising target for the improvement of steroid sensitivity. miRNAs are post-transcriptional regulators of gene expression, which bind to complementary sequences in the target mRNA to induce gene repression $(8,9)$. Previous data indicates that GR $\alpha$-specific miRNAs represent potential therapeutic targets in T-cell acute lymphoblastic leukemia and multiple myeloma $(10,11)$. However, the regulation of GR $\alpha$ by miRNAs in podocytes remains to be determined. In the present study, the miRNA expression profile in normal murine podocytes and injured podocytes induced by puromycin aminonucleoside 
(PAN) was compared, and demonstrated the existence of an association between miR-30a and the expression of GR, based on bioinformatic analysis. Subsequently, the present study observed that miR-30a downregulated GR $\alpha$ in injured podocytes. Furthermore, inhibition of miR-30a reduced podocytic apoptosis by upregulating GR $\alpha$. These findings indicated that inhibition of miR-30a may reduce podocytic apoptosis via a GR $\alpha$-mediated mechanism.

\section{Materials and methods}

miRNA microarray. miRNA microarray analysis was performed using miRNA microarrays (RiboBio Co., Ltd., Guangzhou, China), according to the manufacturer's instructions. Briefly, $600 \mathrm{ng}$ total RNA was isolated from differentiated murine podocytes and injured podocytes induced by PAN, and was dephosphorylated with a calf intestine alkaline phosphatase treatment for $30 \mathrm{~min}$ at $37^{\circ} \mathrm{C}$ prior to labeling The samples were diluted with dimethylsulfoxide, denatured for $10 \mathrm{~min}$ at $100^{\circ} \mathrm{C}$ and were subsequently labeled with pCp-Cy3 (Biorbyt, Cambridge, UK) in T4 RNA ligation buffer (BestBio, Shanghai, China). The labeled RNA was hybridized using 2X hybridization buffer (Agilent Technologies, Palo Alto, CA, USA), washed with 6X SSC (Solarbio Science \& Technology Co., Ltd., Beijing, China)/0.005\% Triton X-102 (DOW, Midland, MI, USA), stained with horseradish peroxidase-streptavidin (RiboBio Co., Ltd.) and scanned with an Agilent 2200 Bioanalyzer (Agilent Technologies). Microarray data analysis was performed using an Illumina HiSeq $^{\mathrm{TM}} 2500$ (Illumina Inc., San Diego, CA, USA).

Prediction of murine GR targeting miRNAs. The following target prediction algorithms were used to predict murine GR targeting miRNAs: Pictar (http://pictar.mdc-berlin. de/), miRanda (http://www.microrna.org/) and TargetScan/TargetScanS (http://www.targetscan.org/). The highest threshold value was used for the prediction using miRanda.

Podocytes culture. The conditionally immortalized mouse MPC5 podocyte cell line was cultured, as previously described (6). Briefly, undifferentiated podocytes were grown at $33^{\circ} \mathrm{C}$ with a relative humidity of $100 \%$ and $5 \% \mathrm{CO}_{2}$-containing atmosphere in RPMI-1640 medium (Hyclone, Logan, UT, USA), supplemented with $10 \%$ fetal bovine serum (FBS; Gibco Life Technologies, Grand Island, NY, USA), 100 U/ml penicillin, $100 \mu \mathrm{g} / \mathrm{ml}$ streptomycin (Proteintech Group, Inc., Chicago, IL, USA) and $10 \mathrm{U} / \mathrm{ml}$ mouse interferon- $\gamma$ (IFN- $\gamma$; Sigma Chemical Co., St. Louis, MO, USA). Podocyte differentiation was induced by culturing at $37^{\circ} \mathrm{C}$ for $10-14$ days in the presence of rat tail collagen type I (Sigma Chemical Co.) without IFN- $\gamma$.

Podocyte treatment. Podocyte injury was induced in vitro with PAN (Sigma Chemical Co.) using the following procedure: Differentiated podocytes (day 12) were incubated in RPMI-1640 medium, supplemented with charcoal-stripped FBS for $24 \mathrm{~h}$. Drug treatments were performed $24 \mathrm{~h}$ following transfection. The podocytes were exposed to PAN at a concentration of $45 \mathrm{ng} / \mathrm{ml}$ and were incubated for $48 \mathrm{~h}$. Podocyte recovery was induced using dexamethasone (Dex; Sigma
Chemcial Co.). The cells were pretreated for $1 \mathrm{~h}$ with the GR agonist, Dex $(1 \mu \mathrm{M})$ prior to PAN treatment. Correspondingly, the cells were pretreated with the GR antagonist, RU486 (20 $\mu \mathrm{M}$; Sigma Chemical Co.), for $30 \mathrm{~min}$ prior Dex treatment.

Transfection. Using the methods described by the relevant manufacturers, the podocytes (day 12) were transiently transfected with $50 \mathrm{nM}$ synthetic miR-30a mimics, $100 \mathrm{nM}$ inhibitor and the corresponding scrambled control oligonucleotides (RiboBio Co., Ltd.). At 48 h post-transfection, the cells were harvested and prepared for analysis.

Total RNA isolation and reverse transcription-quantitative polymerase chain reaction $(R T-q P C R)$. On the 12 th day of podocyte differentiation (day 12), the total RNA was isolated using TRIzol reagent (Invitrogen Life Technologies, Carlsbad, CA, USA), according to the manufacturer's instructions. The RNA concentration and purity was assessed by measuring the absorbance at 260 and $280 \mathrm{~nm}$ using a Nanodrop spectrophotometer 2000 (Thermo Fisher Scientific, Waltham, MA, USA).

The primers for assay of miR-30a were purchased from ABI (Applied Biosystems, Foster City, CA, USA). RT reactions, containing the total RNA $(5 \mu \mathrm{l}), 50 \mathrm{nM}$ stem-loop RT primer $(3 \mu \mathrm{l})$, 10X RT buffer $(1.5 \mu \mathrm{l}), 100 \mathrm{mM}$ dNTPs $(0.15 \mu \mathrm{l})$, multiScribe reverse transcriptase $(1 \mu \mathrm{l})$ and RNase inhibitor $(0.19 \mu \mathrm{l})$, were performed using a GeneAmp PCR system 9700 (Applied Biosystems) for $30 \mathrm{~min}$ at $16^{\circ} \mathrm{C}, 30 \mathrm{~min}$ at $42^{\circ} \mathrm{C}$ and $5 \mathrm{~min}$ at $85^{\circ} \mathrm{C}$. PCR amplification was performed using a standard TaqMan PCR kit (Applied Biosystems) with the RT product $(1.33 \mu \mathrm{l}), 2 \mathrm{X}$ TaqMan Universal PCR Master $\operatorname{mix}(14.5 \mu \mathrm{l}), 20 \mathrm{X}$ TaqMan MicroRNA assay $(1.5 \mu \mathrm{l})$. The PCR amplification conditions were as follows: $95^{\circ} \mathrm{C}$ for $10 \mathrm{~min}$, followed by 40 cycles of $95^{\circ} \mathrm{C}$ for $15 \mathrm{sec}$ and $60^{\circ} \mathrm{C}$ for $1 \mathrm{~min}$. The intra-experimental variation was assessed based on the average of the mean $\Delta \Delta \mathrm{Ct}$ values following normalization against U6. All samples were assessed in triplicate.

The gene expression levels were analyzed by RT-qPCR on a LightCycler (Roche, Mannheim, Germany) using the following primers (Takara Bio, Inc., Otsu, Japan): GR $\alpha$, sense: 5'-AAAGAGCTAGGAAAAGCCATTGTC-3' and antisense: 5'-TCAGCTAACATCTCTGGGAATTCA-3'; GAPDH, sense: 5'-GAAGGTGAAGGTCGGAGT-3' and antisense: 5'-GAA GATGGTGATGGGATTTC-3'. The total RNA $(2 \mu \mathrm{l})$ was used for RT using the $\mathrm{SYBR}^{\circledR}$ PrimerScript $_{\mathrm{TM}}$ RT-PCR kit (Takara Bio, Inc.), according to the manufacturer's instructions. The resulting data were analyzed using the comparative $\Delta \Delta \mathrm{C}_{\mathrm{t}}$ method for relative gene expression quantification against GAPDH (12).

Analysis of the expression of GRa by western blotting. The protein concentration of the cell lysates $(100 \mu \mathrm{g})$ was determined using a bicinchoninic acid protein assay kit (Pierce Biotechnology, Rockford, IL, USA). MPC5 cells grown in six-pore plates (Costar Corning, Corning, NY, USA) were washed twice with PBS, then treated with RIPA cell lysis solution (Solarbio Science \& Technology Co., Ltd.) on ice for $30 \mathrm{~min}$, then centrifuged at $12,000 \mathrm{x} \mathrm{g}$ for $30 \mathrm{~min}$ at $4^{\circ} \mathrm{C}$ to attain the supernatant. The protein samples were heated to $100^{\circ} \mathrm{C}$ for $5 \mathrm{~min}$ prior to $20 \mu \mathrm{l}$ aliquots being separated by SDS-PAGE (Beijing Solarbio Science \& Technology Co., 
Ltd.) and transferred onto polyvinylidene membranes (Beijing Solarbio Science \& Technology Co.,Ltd.). The membranes were incubated with a polyclonal rabbit-anti-mouse GR $\alpha$ primary antibody (cat. no. sc-37642; 1:250; Santa Cruz Biotechnology, Inc., CA, USA) in $10 \mathrm{ml}$ dilution buffer (1X Tris-buffered saline, $0.1 \%$ Tween-20 and 5\% BSA), with agitation overnight at $4^{\circ} \mathrm{C}$. The membranes were subsequently incubated with the appropriate horseradish peroxidase-conjugated anti-rabbit immunoglobulin G (cat. no. zf-0311; 1:2,000; Zhongshan Biotechnology Co., Ltd., Beijing, China) in $10 \mathrm{ml}$ dilution buffer with agitation for $1 \mathrm{~h}$ at $25^{\circ} \mathrm{C}$. The expression of GR $\alpha$ was normalized against GAPDH, as an internal control. The bands were detected using an enhanced chemiluminescence kit (Amersham, Pittsburgh, PA, USA) and the protein expression was quantified using Labworks ${ }^{\mathrm{TM}}$ Image Acquisition and Analysis software version 4.6 (UVP LLC, Upland, CA, USA).

Flow cytometric analysis of podocyte apoptosis. The apoptosis rate in the transfected podocytes exposed to Dex and PAN was assessed using a fluorescein isothiocyanate (FITC)-conjugated annexin V apoptosis detection kit (Molecular Probes, Eugene, Oregon, USA). Briefly, the podocytes were gently trypsinized and pelleted by centrifugation at $900 \mathrm{x} \mathrm{g}$ for $5 \mathrm{~min}$ at room temperature. The pellet was washed with phosphate-buffered saline (PBS) and resuspended in binding buffer. The cells were subsequently stained with FITC-annexin V and propidium iodide (PI) for $5 \mathrm{~min}$ on ice in the dark. The stained cell data was acquired on a FACScan flow cytometer (Becton Dickinson, San Jose, CA, USA) and analyzed using CellQuest Pro software 5.1 (BD Biosciences, San Jose, CA, USA). At least $1 \times 10^{6} / \mathrm{ml}$ cells were assessed per sample and the apoptotic cells were defined as annexin $\mathrm{V}$ positive and PI negative.

Podocyte viability assays. Podocytes transfected with the miR-30a mimics and inhibitor were pretreated with Dex followed by PAN. The viability was determined by fluorescence microscopy (BX51TF; Olympus, Tokyo, Japan) following staining with an EdU incorporation kit (RiboBio Co. Ltd.), according to the manufacturer's instructions.

Cloning of pmiR-RB-REPORT $T^{T M}$ construct. Prediction of the 3'-untranslated region (UTR) and coding sequence (CDS) was performed with miRanda v1.0b and RNA22, respectively. The GR $\alpha$ 3'-UTR (1-2021 bp) fragment, bearing the predicted miR-30a binding site, was isolated from murine cDNA by PCR. The PCR product was isolated by agarose gel electrophoresis, purified and cloned into the pmiR-RB-REPORT ${ }^{\mathrm{TM}}$ vector (RiboBio Co., Ltd.). The resulting construct was designated 3'-UTR (1-2021 bp) WT. The predicted miR-30a binding site (GTTTGTG) was mutated (CTATCTC), inserted into another vector and designated 3'-UTR (1-2021 bp) MT. The GR $\alpha$ 3'-UTR (2020-3953 bp) and CDS fragments were cloned into another two vectors, and were designated 3'-UTR (2020-3953 bp) WT and CDS WT, respectively. The primer sequences (RiboBio Co., Ltd.) were as follows: 3'-UTR (1-2021 bp) WT and 3'-UTR (1-2021 bp) MT, sense: 5'-CCGCTCGAGCTGCCTTACTAAGAAAGG CTGCCTTAAAG-3' and antisense: 5'-GAATGCGGCCGC AGCCTCTGTTTCTGTTAACC-3'; 3'-UTR (2020-3953 bp) WT, sense: 5'-CCGCTCGAGACAAGAGTCCATTTCCAA
GTA AG-3' and antisense: 5'-GAATGCGGCCGCAGT CACATAATGAAAAAGCAG-3'; CDS WT, sense: 5'-CCG CTCGAGATGGACTCCAAAGAATCCTTAGC-3' and antisense: 5'-GAATGCGGCCGCTCATTTCTGATGAAA CAGAAGC-3'. The plasmid constructs were confirmed by automated DNA sequencing.

Luciferase assays. NIH3T3 murine fibroblast cells were seeded into a 96-well plate $(4,000$ cells/well) and were co-transfected with a reporter construct $(100 \mathrm{ng} / \mathrm{ml})$ and synthetic miR-30a (50 $\mathrm{nM}$ ), according to the manufacturer's instructions (RiboBio Co. Ltd.). At $24 \mathrm{~h}$ post-transfection, the cells were harvested in $100 \mu 1$ Passive Lysis Buffer (Promega, Madison, WI, USA) and the luciferase activity was measured in the lysates (35 $\mu \mathrm{l})$ using a luciferase reporter assay kit (Promega).

Statistical analysis. SPSS version 18.0 (IBM SPSS, Chicago, IL, USA) was used for statistical analyses. For microarray analysis, the raw expression signals were log transformed, normalized and filtered, according to the median corrected signal of all the miRNAs with an intensity $>100$. The miRNAs displaying differential expression between differentiated murine podocytes and injured podocytes induced by PAN were detected using a two-sample t-test. The data are presented as the mean \pm standard deviation and interpreted by analysis of variance with Tukey's test. $\mathrm{P}<0.05$ was considered to indicate a statistically significant difference.

\section{Results}

Identification of differentially expressed miRNA in differentiated murine podocytes and injured podocytes induced by $P A N$. Of the 1,908 murine miRNAs represented on the microarrays, 187 were expressed in differentiated podocytes and 172 in injured podocytes induced by PAN. A total of 19 miRNAs were identified to be significantly upregulated and 35 were significantly downregulated (Table I). Following the application of a fold change threshold $>2$ (false discovery rate $<0.01$ ), 10 miRNAs, including miR-30a, miR-27a-3p, miR-30d, miR-100, miR181c, miR-5099, miR-3535, miR-140-3p, miR-148-3p and miR-103-3p were significantly upregulated.

GR is a putative target of miR-30a in murine podocytes. To identify miRNAs with the potential to modify GR activity by binding to the 3'-UTR in the 10 significantly upregulated miRNAs, an in silico search was performed using Pictar, miRanda and TargetScan/TargetScanS databases. Based on the maximum likelihood of binding conservation and accessibility, all three databases predicted that GR was a putative target of miR-30a in the mouse.

miR-30a negatively regulates GR $\alpha$ in normal and injured podocytes induced by PAN. Based on the results of the bioinformatics investigations, the expression levels of GR $\alpha$ and miR-30a were analyzed in undifferentiated and differentiated podocytes by RT-qPCR. While the expression of miR-30a increased, the expression of GR $\alpha$ decreased during the process of podocytic maturation (Fig. 1A and B). Subsequently, it was revealed that transfection of undifferentiated and differentiated podocytes with the miR-30a mimics caused a reduction in the mRNA 
Table I. Identification of differentially expressed miRNA in differentiated murine podocytes and injured podocytes induced by PAN.

\begin{tabular}{|c|c|c|c|}
\hline miRNA ID & Differentiated podocytes & Podocytes induced by PAN & P-value \\
\hline mmu-miR-30a-5p & 8928.94 & 33333.19 & $<0.01$ \\
\hline mmu-miR-27a-3p & 5597.11 & 18809.07 & $<0.01$ \\
\hline mmu-miR-30d-5p & 4084.54 & 10617.27 & $<0.01$ \\
\hline mmu-miR-100-5p & 576.49 & 2146.32 & $<0.01$ \\
\hline mmu-miR-140-3p & 472.84 & 1605.43 & $<0.01$ \\
\hline mmu-miR-103-3p & 343.27 & 3171.62 & $<0.01$ \\
\hline mmu-miR-148a-3p & 228.49 & 1193.20 & $<0.01$ \\
\hline mmu-miR-181c-5p & 23.17 & 2069.71 & $<0.01$ \\
\hline mmu-miR-5099 & 0.00 & 28.84 & $<0.01$ \\
\hline mmu-miR-3535 & 0.00 & 12.11 & $<0.01$ \\
\hline mmu-miR-10b-5p & 9607.71 & 23206.82 & $<0.05$ \\
\hline mmu-miR-10a-5p & 8993.11 & 22036.14 & $<0.05$ \\
\hline mmu-miR-106b-5p & 359.89 & 881.80 & $<0.05$ \\
\hline mmu-miR-28a-3p & 89.02 & 252.70 & $<0.05$ \\
\hline mmu-miR-542-3p & 31.25 & 98.28 & $<0.05$ \\
\hline mmu-miR-195a-5p & 3.81 & 22.69 & $<0.05$ \\
\hline mmu-miR-429-3p & 3.35 & 20.99 & $<0.05$ \\
\hline mmu-miR-6236 & 0.00 & 7.00 & $<0.05$ \\
\hline mmu-miR-26a-5p & 5669.51 & 376.40 & $<0.01$ \\
\hline mmu-miR-107-3p & 1315.63 & 81.05 & $<0.01$ \\
\hline mmu-miR-378d & 639.75 & 0.00 & $<0.01$ \\
\hline mmu-miR-128-3p & 574.66 & 72.69 & $<0.01$ \\
\hline mmu-miR-181b-5p & 478.94 & 215.67 & $<0.01$ \\
\hline mmu-miR-425-5p & 458.21 & 126.43 & $<0.01$ \\
\hline mmu-miR-22-3p & 408.67 & 95.38 & $<0.01$ \\
\hline mmu-miR-191-5p & 403.18 & 144.69 & $<0.01$ \\
\hline mmu-miR-3074-2-3p & 323.61 & 142.13 & $<0.01$ \\
\hline mmu-miR-29b-3p & 172.70 & 77.29 & $<0.01$ \\
\hline mmu-miR-423-3p & 124.54 & 58.70 & $<0.01$ \\
\hline mmu-miR-615-3p & 115.54 & 39.59 & $<0.01$ \\
\hline mmu-miR-16-1-3p & 87.19 & 11.77 & $<0.01$ \\
\hline mmu-miR-25-5p & 74.39 & 7.85 & $<0.01$ \\
\hline mmu-miR-362-5p & 64.94 & 2.39 & $<0.01$ \\
\hline mmu-miR-671-5p & 51.52 & 5.29 & $<0.01$ \\
\hline mmu-miR-450a-5p & 33.69 & 16.72 & $<0.01$ \\
\hline mmu-miR-296-3p & 29.57 & 13.31 & $<0.01$ \\
\hline mmu-miR-24-3p & 26.98 & 0.00 & $<0.01$ \\
\hline mmu-miR-340-3p & 23.78 & 5.12 & $<0.01$ \\
\hline mmu-miR-24-2-5p & 17.83 & 6.48 & $<0.01$ \\
\hline mmu-miR-30c-2-3p & 14.94 & 5.97 & $<0.01$ \\
\hline mmu-miR-152-5p & 14.63 & 3.92 & $<0.01$ \\
\hline mmu-let-7d-3p & 14.48 & 5.46 & $<0.01$ \\
\hline mmu-miR-350-5p & 14.33 & 3.58 & $<0.01$ \\
\hline mmu-miR-126a-3p & 10.21 & 3.58 & $<0.01$ \\
\hline mmu-miR-199b-3p & 10.21 & 0.00 & $<0.01$ \\
\hline mmu-miR-466e-3p & 10.06 & 0.00 & $<0.01$ \\
\hline mmu-miR-132-5p & 6.25 & 0.00 & $<0.01$ \\
\hline mmu-miR-301a-3p & 14.18 & 7.00 & $<0.05$ \\
\hline mmu-miR-1306-3p & 7.77 & 2.05 & $<0.05$ \\
\hline mmu-miR-182-3p & 6.25 & 2.05 & $<0.05$ \\
\hline mmu-miR-181b-1-3p & 6.10 & 0.00 & $<0.05$ \\
\hline mmu-miR-3102-3p.2-3p & 5.79 & 0.00 & $<0.05$ \\
\hline mmu-miR-1964-3p & 5.03 & 0.00 & $<0.05$ \\
\hline
\end{tabular}

PAN, puromycin aminonucleoside; miRNA, microRNA; miR, microRNA. 


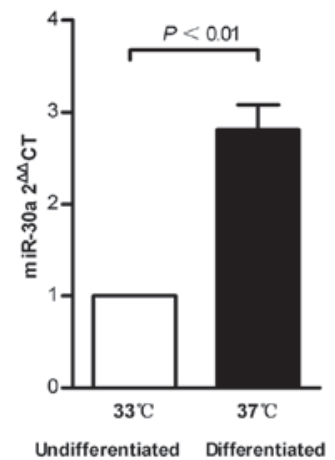

B

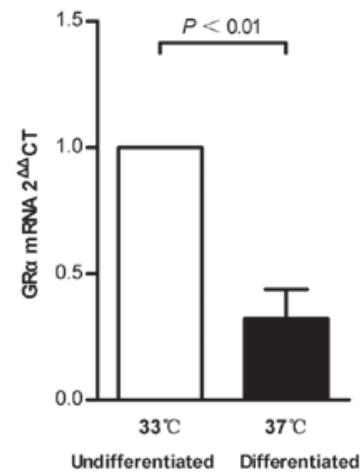

E
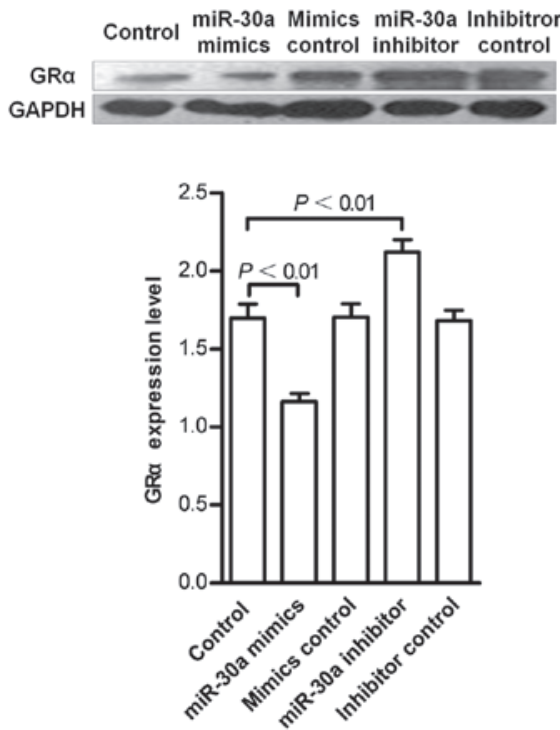

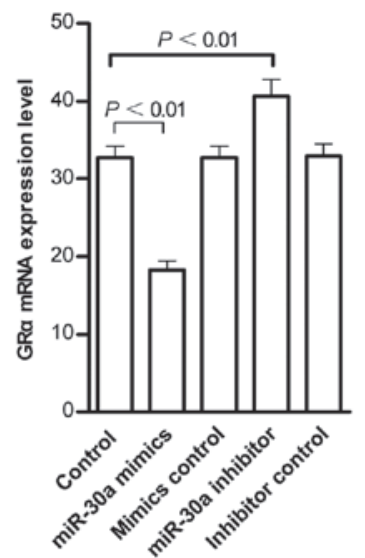

D

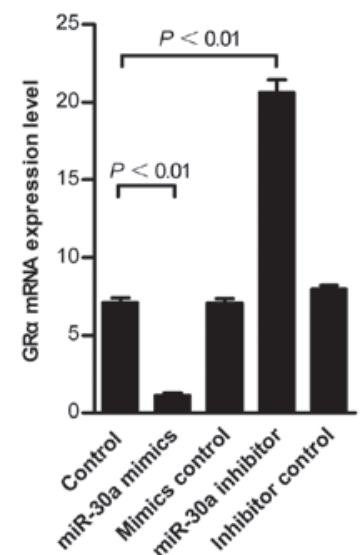

$\mathbf{F}$
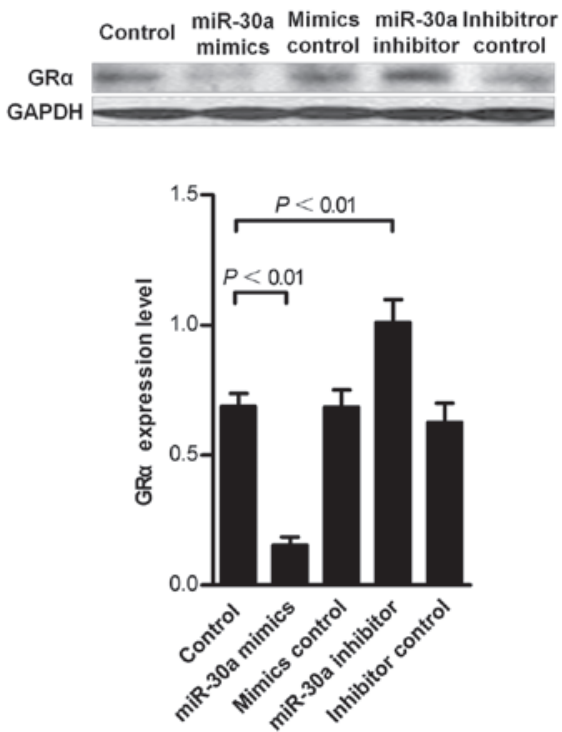

Figure 1. miR-30a negatively regulates GR $\alpha$ in undifferentiated and differentiated murine podocytes. RT-qPCR analysis of the mRNA expression levels of (A) miR-30a and (B) GR $\alpha$ in undifferentiated and differentiated podocytes. RT-qPCR analysis of the mRNA expression of GR $\alpha$ in podocytes transfected with miR-30a mimics or inhibitor in (C) undifferentiated and (D) differentiated podocytes. Western blot analysis of the protein expression of GR $\alpha$ in podocytes transfected with miR-30a mimics or inhibitor in (E) undifferentiated and (F) differentiated podocytes. GAPDH was used as a loading control. The data are expressed as the mean \pm standard deviation of three independent experiments. GR, glucocorticoid receptor; RT-qPCR, reverse transcription-quantitative polymerase chain reaction; miR, microRNA.

and protein expression levels of GR $\alpha$ (25-60 and 25-40\%, respectively). By contrast, treatment with the miR-30a inhibitor resulted in increased mRNA and protein expression levels of GR $\alpha$ (25-35 and 10-25\%, respectively; Fig. 2C-F).

An in vitro model of podocyte injury induced by PAN was established to further confirm the effect of miR-30a on GR $\alpha$. Compared with the normal group, the mRNA expression of GR $\alpha$ decreased in injured podocytes and the expression of miR-30a increased, which confirmed the microarray results (Fig. 2A and B). An identical pattern was observed in response to PAN-injury following miR-30a mimic or inhibitor transfection of the podocytes. Transfection with the miR-30a mimics caused a $25 \%$ reduction in the mRNA and protein expression levels of GR $\alpha$, while miR-30a inhibitor treatment resulted in a $25 \%$ increase in GR $\alpha$ RNA, and a $10 \%$ increase in GR $\alpha$ protein (Fig. 2C-E).

Inhibition of miR-30a prevents PAN-induced podocyte apoptosis. Apoptosis is the predominant characteristic of
PAN-induced podocytic injury. Flow cytometric analysis revealed that podocyte apoptosis increased following PAN treatment and was ameliorated by Dex $(\mathrm{P}<0.05)$. However, this amelioration was inhibited by the GR inhibitor, RU486 (P>0.05). Transfection of PAN and Dex pretreated podocytes with miR-30a mimics or the miR-30a inhibitor resulted in increased and decreased levels of apoptosis, respectively $(\mathrm{P}<0.05$; Fig. 3A and $\mathrm{B})$.

Furthermore, EdU incorporation analysis of the injured podocytes demonstrated that the proliferation rate was increased by Dex and that this increase was inhibited by RU486 $(\mathrm{P}<0.05)$. Transfection of PAN and Dex pretreated podocytes with miR-30a mimics or the miR-30a inhibitor resulted in decreased $(\mathrm{P}<0.05)$ and increased proliferation, respectively (Fig. 3C).

Expression of GR $\alpha$ is downregulated by miR-30a interference, however, the 3'-UTR and CDS of GR $\alpha$ are indirect miR-30a targets. To further confirm the association between miR-30a 
A

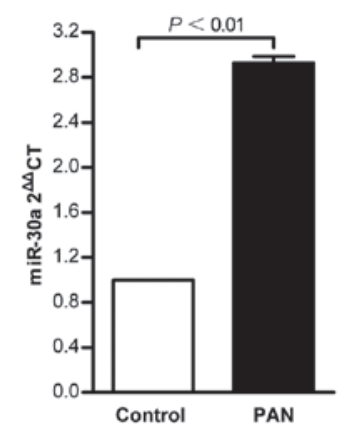

B

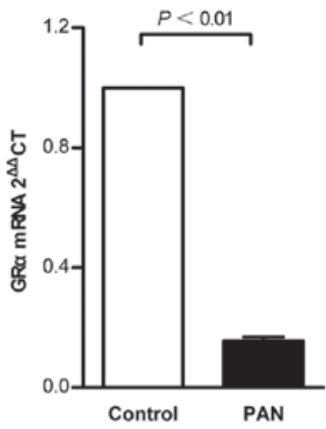

C

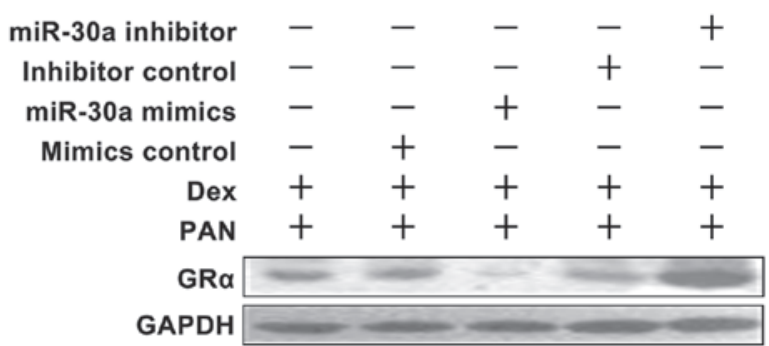

$\mathbf{E}$

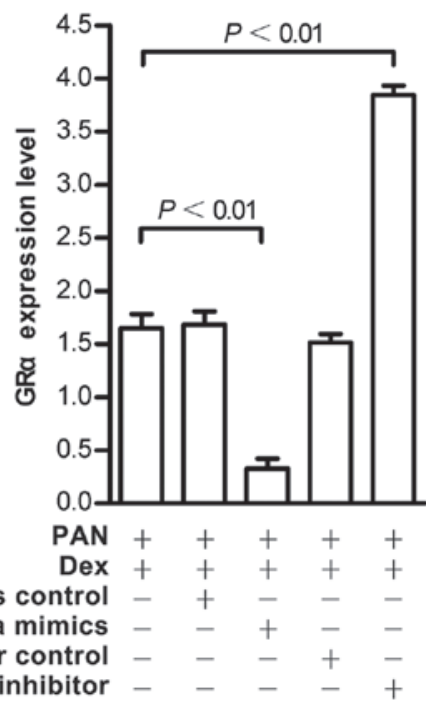

Figure 2. miR-30a negatively regulates GR $\alpha$ in PAN-injured podocytes. RT-qPCR analysis of the mRNA expression levels of (A) miR-30a and (B) GR $\alpha$ in PAN-injured podocytes. (C) RT-qPCR analysis of the mRNA expression of GR $\alpha$ in podocytes transfected with miR-30a mimics and inhibitor prior to PAN-mediated injury. (D and E) Western blot analysis of the protein expression of GR $\alpha$ in podocytes transfected with miR-30a mimics or inhibitor prior to PAN-mediated injury. GAPDH was used as a loading control. The data are expressed as the mean \pm standard deviation of three independent experiments. GR, glucorticoid receptor; PAN, puromycin aminonucleoside; RT-qPCR, reverse transcription-quantitative polymerase chain reaction; miR, microRNA; Dex, dexamethasone.

and GR $\alpha$, the targeted binding site was examined using a dual-luciferase reporter system. Firstly, the consensus sequences for miR-30a in the most common targeted binding site, the GR $\alpha$ 3'-UTR, were predicted. The full-length GR $\alpha$ 3'-UTR (3,953 bp) was revealed to contain six predicted binding sites for miR-30a: 330-351, 741-761, 824-842, 941-969, 1605-1629 and 2108-2137 bp (Fig. 4A). The site located at $941-969$ bp was identified as the most likely binding site, according to the continuity of seed sequences, the lowest free energy and conservation between species. To confirm the predicted consensus sequences and to determine whether these miR-30a seed sequences directly contributed to the negative regulation of GR $\alpha$ expression, substitution mutants (at positions 963, 965,967 and 969) of the GR $\alpha$ 3'-UTR fragment were cloned into vectors containing the WT miR-30a targeting sequences. Furthermore, analysis of other possible candidates, including the GR $\alpha-5$ '-UTR and CDS, indicated that nucleotides 66-71 bp of the CDS region is also a predicted miR-30a binding consensus sequence (Fig. 4B). However, co-transfection of murine cells with $50 \mathrm{nM}$ miR-30a mimics revealed no change in luciferase activity of the vectors, therefore, demonstrating that miR-30a binds to either the 3'-UTR or CDS of GR $\alpha$ (Fig. 4C).

\section{Discussion}

$\mathrm{GR} \alpha$ is a well-known receptor isoform, which mediates glucocorticoid functions. Investigation of the miRNA-regulation of GR $\alpha$ in podocytes may shed new light on the mechanisms underlying steroid-resistance in podocyte-associated nephrology. By comparing a microarray miRNA expression profile in normal and injured murine podocytes, the present study identified 10 candidate miRNAs significantly upregulated in injured podocytes, along with the downregulation of their potential target gene, GR $\alpha$.

Since a certain regularity exists in the interaction of miRNAs and target genes, computational algorithms, which are fundamental to our understanding of the role of miRNAs in gene regulation, have proved to be highly effective in predicting candidate miRNAs, which can target an mRNA of interest $(13,14)$. However, this predicted correlation requires biological confirmation in the context of several influencing factors (15). The three most common bioinformatics target prediction tools were used in the present study and all indicated that GR was putative target gene of miR-30a. However, the interaction between miR-30a and the GR $\alpha$ isoform of GR 
A

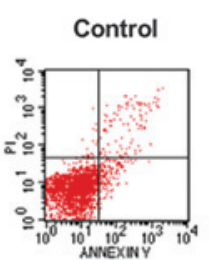

PAN+Dex
+ miR-30a mimic

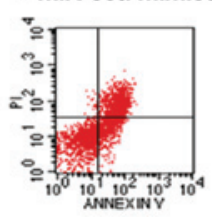

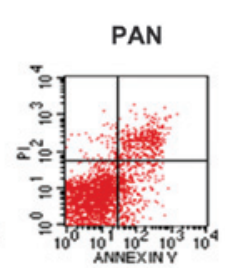

PAN+Dex + mimics contro

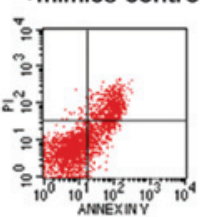

PAN+Dex

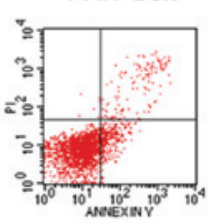

PAN+Dex
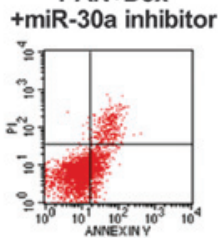

B

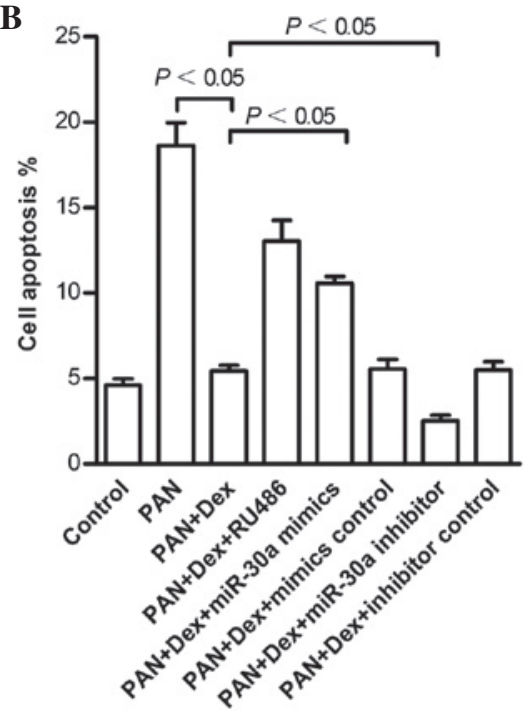

C

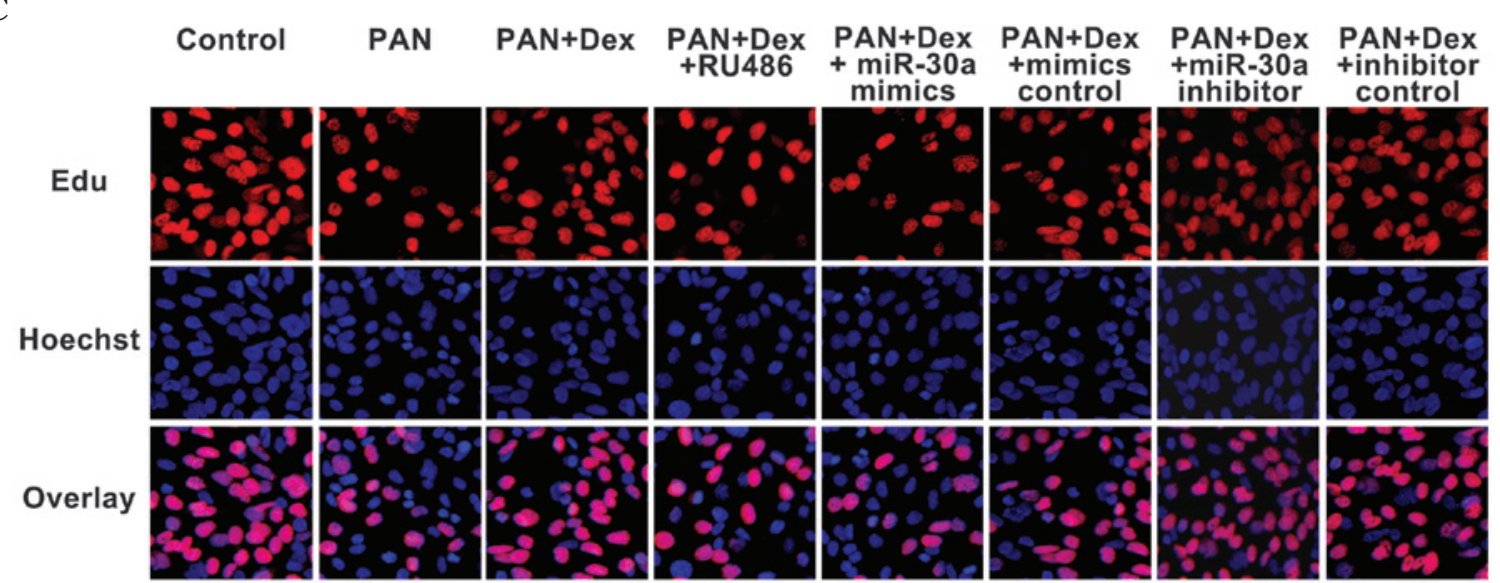

Figure 3. Inhibition of miR-30a prevents PAN-induced podocytic apoptosis. (A and B) Flow cytometric analysis of the apoptotic cell death marker, annexin V, in PAN-injured podocytes following recovery induced by Dex combined with GR antagonist, RU486, and following transfection with miR-30a mimics and the inhibitor. The data are expressed as the mean \pm standard deviation of three independent experiments. (C) EdU incorporation was used to observe cell proliferation. Dex, dexamethasone; GR, glucorticoid receptor; PAN, puromycin aminonucleoside; miR, microRNA.

remains to be elucidated experimentally. As classical negative regulators in mammals, miRNAs were initially hypothesized to mediate the repression of target proteins at the translational level (16). Later, high-throughput studies confirmed that miRNA-mediated changes occur at the mRNA and protein levels, and are predominantly mediated by target mRNA destabilization $(17,18)$. In the present study, it was identified that the marked reduction in the mRNA expression of GR $\alpha$ mediated by miR-30a was in contrast to the more moderate influence on the corresponding protein, indicating that miR-30a regulates GR $\alpha$ expression at the translational and transcriptional levels.

The podocyte is a terminally differentiated cell with limited proliferative capacity and podocyte number is a hallmark of progressive glomerulosclerosis $(19,20)$. Therefore, podocytic apoptosis is a critical determinant of the progression of glomerulosclerosis and protection against podocytic apoptosis is a vital means of slowing down glomerulosclerosis (21). Additionally, previous studies have suggested that miR-30a is involved in kidney development, including podocyte differentiation (22-24). Therefore, the present study aimed to determine whether miR-30a was involved in podocytic apoptosis. Besides the conventional anti-inflammatory or immunosuppressive actions, Dex causes modest upregulation and increased nuclear localization of GR in podocytes, and also enhances and accelerates podocytic maturation. PAN treatment causes podocytic apoptosis, resulting in the development of massive proteinuria resembling human podocytopathies. This method has provided insights into the mechanisms of podocyte-induced injury in vitro. Glucocorticoids can protect and enhance recovery from PAN-induced injury in cultured murine podocytes via actin filament stabilization (7). The protective effects of Dex on PAN-induced podocyte apoptosis are associated with decreased expression of $\mathrm{p} 53$, increased expression of $\mathrm{Bcl}-\mathrm{xl}$, inhibition of apoptosis-inducing factor translocation and ERK phosphorylation (25-27). This also remains controversial as certain previous studies have demonstrated that Dex significantly enhances podocyte number owing to increased survival of individual cells as opposed to a reduction of apoptosis (4). To confirm the microarray results, quantitative detection of miR-30a and the expression was higher in the injured podocytes induced by PAN, confirming the microarray results. However, Wu et al (28) previously documented that the expression of the miR-30 family in human 


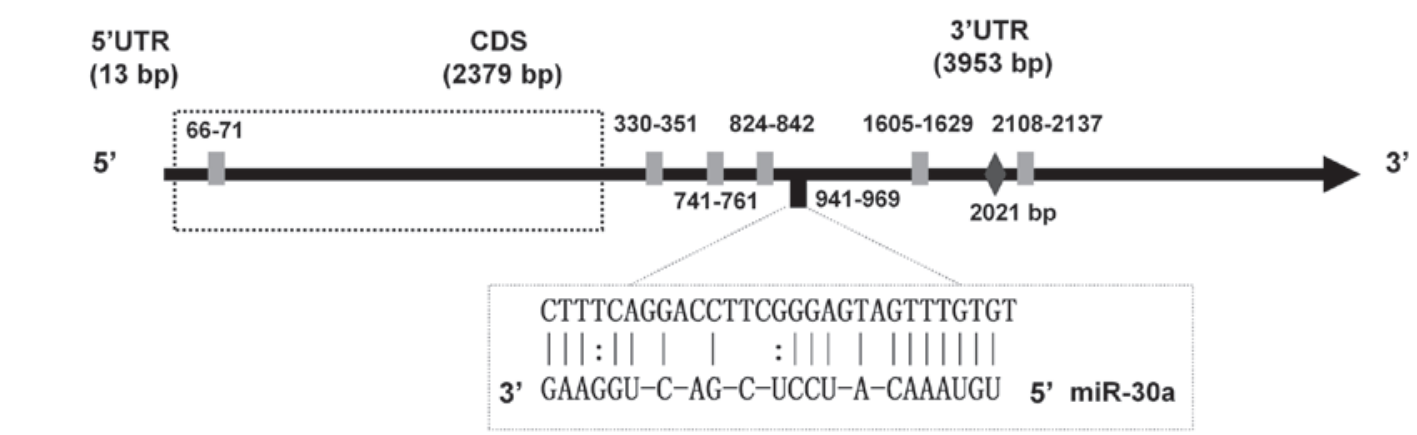

B

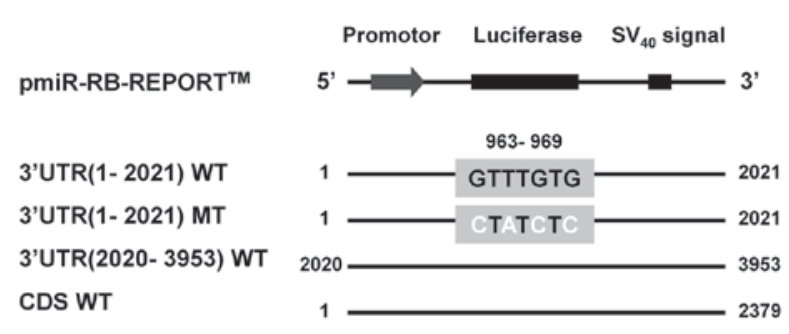

C

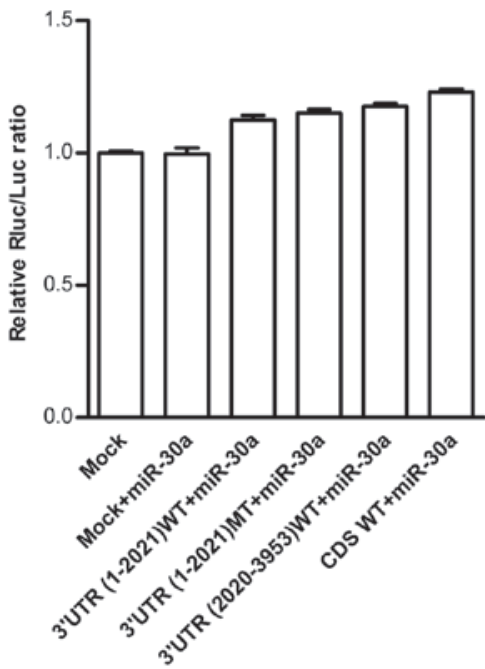

Figure 4. Expression of GR $\alpha$ is downregulated by miR-30a interference, however the 3'-UTR and CDS of GR $\alpha$ are indirect miR-30a targets. (A) Predicted miR-30a target sites within the full-length murine GR $\alpha$ mRNA sequence were identified using TargetScan 5.1 software. The putative miRNA target sequences are located at the 3'-UTR and CDS. (B) Construction of luciferase reporters containing the full-length GR $\alpha$ CDS and 3'-UTR, which included the miR-30 binding sites, and harboring mutations at 963-969 bp. (C) Co-transfection with miR-30a mimics resulted in no change in the luciferase activity of all of vectors. The data are presented as the mean \pm standard deviation of three independent experiments. UTR, untranslated region; GR, glucocorticoid receptor; miR, microRNA; WT, wild-type; MT, mutant; CDS, coding sequence.

podocytopathies were downregulated. The present study hypothesized that this may be associated with the tissue and temporal specificity of miRNA. Therefore, the present study investigated whether GR $\alpha$ was indeed regulated by miR-30a and whether the observed reduction in podocyte apoptosis, and upregulation in the expression of GR $\alpha$ following PAN-induced injury, were mediated by miR-30a. These findings demonstrated that transfection of miR-30a exerted a negative-regulatory effect on the expression of GR $\alpha$ in PAN-injured podocytes. In the present study, Dex was used at $1 \mu \mathrm{M}$, which is consistent with clinical doses of glucocorticoid used in the treatment of glomerular diseases and mimics the in vivo therapeutic levels $(4,5)$. Dex reduced PAN-induced podocytic apoptosis and this effect was inhibited by the GR antagonist, RU486. Therefore, it was suggested that the therapeutic effect of Dex was GR-dependent. In addition, inhibition of miR-30a in podocytes upregulated the expression of GR $\alpha$, reduced apoptosis and promoted proliferation, therefore, improving podocytic sensitivity to Dex and favoring the therapeutic effect of steroids on podocyte injury.

To further elucidate the direct interaction between miR-30 and GR $\alpha$, computational methods were used to predict the miR-30a binding sites within the GR $\alpha$ mRNA sequence.
Previous studies have demonstrated that miRNAs modulate their target mRNAs in mammalian cells by base pairing with complementary sites commonly located within their 3'-UTR (29,30). Previously, it was demonstrated that miRNA binding sites also exist within the CDS and 5'-UTR $(31,32)$. Computational analysis predicted six potential binding sites for miR-30a within the GR $\alpha$ 3'-UTR sequence, of which the site at 941-969 bp was the most likely based on complementary base pairing, thermodynamic stability or conservation of seed matches. In addition, the programs predicted a potential binding site within the CDS. Luciferase reporters containing the full-length GR $\alpha$ CDS and 3'-UTR, which included the predicted miR-30 binding sites and harboring mutations at 963-969 bp, were generated. However, the luciferase reporter assay data suggested an indirect effect on GR $\alpha$ transcriptional activity since miR-30a failed to repress luciferase activity. Since animal miRNAs have limited sequence complementarity to their targets, it is a challenging task to predict miRNA targets with high precision. In order to reduce the proportion of false positives obtained through in silico prediction, the use of $>3$ of the prediction programs is recommended for this purpose, although Selbach et al $(33,34)$ reported that this approach is associated with precision levels of only about $60 \%$. Several novel 
computational target prediction programs were identified to achieve precision reaching $\sim 93 \%(35,36)$. Accordingly, false positives obtained through in silico prediction may account for the negative result observed in the luciferase reporter assay in the present study. However, the correlation between miR-30a and GR $\alpha$ at the functional level was demonstrated in this study. A similar effect was reported by Vreugdenhil et al (37) and revealed that miR-18 was predicted to bind GR mRNA in silico and that miR-18 overexpression reduced GR protein levels. However, miR-18 was unable to bind to the predicted seed region in the GR 3'-UTR. It was suggested that the predicted miRNA binding sites were unavailable for miRNA binding due to differences in the tertiary RNA folding of the target gene sequence in the predicted and in vivo situations (37). Therefore, these data fail to completely preclude a potential miR-30a effect on the gene expression of GR $\alpha$. The functions of miRNAs are particularly complicated. While a single miRNA is thought to target numerous genes, multiple miRNAs are co-expressed and are likely to mediate coordinated regulation of identical mRNAs $(28,38)$, resulting in corresponding multiplex associations. Furthermore, synergy and antagonism also exist in the interaction of miRNAs and represent an additional layer of diversity to the function of miRNAs (39). The mechanisms by which miRNAs mediate gene regulation are still open to discussion, and further investigations are required to improve our understanding of the function of each miRNA. Only in this way will the network of interactions, which exist between miRNAs and target genes, be better elucidated.

The major finding of the present study was that silencing of miR-30a may have a potential effect on improving steroid sensitivity in murine podocyte injury. However, although miR-30a downregulates GR $\alpha$ at the functional level, the mechanism requires further investigation.

\section{Acknowledgements}

This study was supported by a grant from the General Program of National Natural Science Foundation of China (grant no. 81200522).

\section{References}

1. Pollak MR: Inherited podocytopathies: FSGS and nephrotic syndrome from a genetic viewpoint. J Am Soc Nephrol 13: 3016-3023, 2002.

2. Chuang PY and He JC: Signaling in regulation of podocyte phenotypes. Nephron Physiol 111: p9-p15, 2009.

3. Rhen T and Cidlowski JA: Anti-inflammatory action of glucocorticoids-new mechanisms for old drugs. N Engl J Med 353: 1711-1723, 2005.

4. Xing CY, Saleem MA, Coward RJ, Ni L, Witherden IR and Mathieson PW: Direct effects of dexamethasone on human podocytes. Kidney Int 70: 1038-1045, 2006.

5. Guess A, Agrawal S, Wei CC, Ransom RF, Benndorf R and Smoyer WE: Dose-and time-dependent glucocorticoid receptor signaling in podocytes. Am J Physiol Renal Physiol 14: F845-F853, 2010.

6. Ransom RF, Lam NG, Hallett MA, Atkinson SJ and Smoyer WE: Glucocorticoids protect and enhance recovery of cultured murine podocytes via actin filament stabilization. Kidney Int 68 : 2473-2483, 2005.

7. Xie H, Lin HL, Chen JL, et al: Correlation of expression of glucocorticoid receptor $\alpha$ and $\beta$ in podocytes with sensitivity of glucocorticoid in primary nephrotic syndrome. China J Mod Med 36: 35-39, 2012.
8. Lin SL, Chang D, Wu DY and Ying SY: A novel RNA splicingmediated gene silencing mechanism potential for genome evolution. Biochem Biophys Res Commun 10: 754-760, 2003.

9. Shruti K, Shrey K and Vibha R: Micro RNAs: Tiny sequences with enormous potential. Biochem Biophys Res Commun 407: 445-449, 2011.

10. Lv M, Zhang X, Jia H, Li D, Zhang B, Zhang H, Hong M, Jiang T, Jiang Q, Lu J, et al: An oncogenic role of miR-142-3p in human T-cell acute lymphoblastic leukemia (T-ALL) by targeting glucocorticoid receptor- $\alpha$ and cAMP/PKA pathways. Leukemia 26: 769-777, 2012.

11. Tessel MA, Benham AL, Krett NL, Rosen ST and Gunaratne PH: Role for microRNAs in regulating glucocorticoid response and resistance in multiple myeloma. Horm Cancer 2: 182-189, 2011.

12. Livak KJ and Schmittgen TD: Analysis of relative gene expression data using real-time quantitative PCR and the (-Delta Delta C(T)) Method. Methods 25: 402-408, 2001.

13. Sethupathy P, Megraw M and Hatzigeorgiou A: A guide through present computational approaches for the identification of mammalian microRNA targets. Nat Methods 3: 881-886, 2006

14. Wang X: Computational prediction of microRNA targets. Methods Mol Biol 667: 283-295, 2010.

15. Ørom UA and Lund AH: Experimental identification of microRNA targets. Gene 451: 1-5, 2010.

16. Humphreys DT, Westman BJ, Martin DI and Preiss T: MicroRNAs control translation initiation by inhibiting eukaryotic initiation factor 4E/cap and poly(A) tail function. Proc Natl Acad Sci USA 102: 16961-16966, 2005.

17. Guo H, Ingolia NT, Weissman JS and Bartel DP: Mammalian microRNAs predominantly act to decrease target mRNA levels. Nature 466: 835-840, 2010.

18. Baek D, Villén J, Shin C, Camargo FD, Gygi SP and Bartel DP: The impact of microRNAs on protein output. Nature 455: 64-71, 2008.

19. Kriz W, Elger M, Nagata M, Kretzler M, Uiker S, Koeppen-Hageman I, Tenschert S and Lemley KV: The role of podocytes in the development of glomerular sclerosis. Kidney Int 45 (Suppl): S64-S72, 1994.

20. Kriz W, Gretz N and Lemley KV: Progression of glomerular diseases: Is the podocyte the culprit? Kidney Int 54, 687-697, 1998.

21. Kim YH, Goyal M, Kurnit D, Wharram B, Wiggins J, Holzman L, Kershaw D and Wiggins R: Podocyte depletion and glomerulosclerosis have a direct relationship in the PAN-treated rat. Kidney Int 60: 957-968, 2001.

22. Nagalakshmi VK, Ren Q, Pugh MM, Valerius MT, McMahon AP and Yu J: Dicer regulates the development of nephrogenic and ureteric compartments in the mammalian kidney. Kidney Int 385: 1-14, 2010

23. Agrawal R, Tran U and Wessely O: The miR-30 miRNA family regulates Xenopus pronephros development and targets the transcription factor Xlim1/Lhx1. Development 136: 3927-3936, 2009.

24. Harvey SJ, Jarad G, Cunningham J, Goldberg S, Schermer B, Harfe BD, McManus MT, Benzing $\mathrm{T}$ and Miner JH: Podocyte-specific deletion of dicer alters cytoskeletal dynamics and causes glomerular disease. J Am Soc Nephrol 19: 2150-2158, 2008.

25. Wada T, Pippin JW, Nangaku M and Shankland SJ: Dexamethasone's prosurvival benefits in podocytes require extracellular signal-regulated kinase phosphorylation. Nephron Exp Nephrol 109: e8-e19, 2008.

26. Koshikawa M, Mukoyama M, Mori K, Suganami T, Sawai K, Yoshioka T, Nagae T, Yokoi H, Kawachi H, Shimizu F, et al: Role of p38 mitogen-activated protein kinase activation in podocyte injury and proteinuria in experimental nephrotic syndrome. J Am Soc Nephrol 16: 2690-2701, 2005.

27. Wada T, Pippin JW, Marshall CB, Griffin SV and Shankland SJ: Dexamethasone prevents podocyte apoptosis induced by puromycin aminonucleoside: Role of p53 and Bcl-2-related family proteins. J Am Soc Nephrol 16: 2615-2625, 2005.

28. Wu J, Zheng C, Fan Y, Zeng C, Chen Z, Qin W, Zhang C, Zhang W, Wang X, Zhu X, et al: Downregulation of microRNA-30 facilitates podocyte injury and is prevented by glucocorticoids. J Am Soc Nephrol 25: 92-104, 2014.

29. Carthew RW and Sontheimer EJ: Origins and Mechanisms of miRNAs and siRNAs. Cell 136: 642-655, 2009.

30. Fabian MR, Sonenberg N and Filipowicz W: Regulation of mRNA translation and stability by microRNAs. Annu Rev Biochem 79: 351-379, 2010 . 
31. Roberts AP, Lewis AP and Jopling CL: miR-122 activates hepatitis $C$ virus translation by a specialized mechanism requiring particular RNA components. Nucleic Acids Res 39: 7716-7729, 2011.

32. Schnall-Levin M, Zhao Y, Perrimon N and Berger B: Conserved microRNA targeting in Drosophilia is as widespread in coding regions as in 3'-UTRs. Proc Natl Acad Sci USA 107: 15751-15756, 2010.

33. Selbach M, Schwanhäusser B, Thierfelder N, Fang Z, Khanin R and Rajewsky N: Widespread changes in protein synthesis induced by microRNAs. Nature 455: 58-63, 2008

34. Maragkakis M, Alexiou P, Papadopoulos GL, Reczko M, Dalamagas T, Giannopoulos G, Goumas G, Koukis E, Kourtis K, Simossis VA, et al: Accurate microRNA target prediction correlates with protein repression levels. BMC Bioinformatics 10 : $295,2009$.
35. Reyes-Herrera PH, Ficarra E, Acquaviva A and Macii E: miREE: miRNA recognition elements ensemble. BMC Bioinformatics 12: 454, 2011.

36. Mapleson D, Moxon S, Dalmay T and Moulton V: MirPlex: A tool for identifying miRNAs in high-throughput sRNA datasets without a genome. J Exp Zool B Mol Dev Evol 320: 47-56, 2013.

37. Vreugdenhil E, Verissimo CS, Mariman R, Kamphorst JT, Barbosa JS, Zweers T, Champagne DL, Schouten T, Meijer OC, de Kloet ER, et al: MicroRNA 18 and 124a down-regulate the glucocorticoid receptor: Implications for glucocorticoid responsiveness in the brain. Endocrinology 150: 2220-2228, 2009.

38. Bartel DP: MicroRNAs: Target recognition and regulatory functions. Cell 136: 215-233, 2009.

39. Breving $\mathrm{K}$ and Esquela-Kerscher $\mathrm{A}$ : The complexities of microRNA regulation: mirandering around the rules. Int J Biochem Cell Biol 42: 1316-1329, 2010. 